\title{
Need of education on biosimilars amongst ophthalmologists: combating the nocebo effect
}

\author{
Ashish Sharma $^{1}$ - Nilesh Kumar $\mathbb{D}^{1} \cdot$ Francesco Bandello $^{2}$ - Anat Loewenstein ${ }^{3}$ - Baruch D. Kuppermann ${ }^{4}$
}

Received: 4 November 2019 / Revised: 12 November 2019 / Accepted: 19 November 2019 / Published online: 29 November 2019

(c) The Royal College of Ophthalmologists 2019

Biologics are any medicinal products containing biotechnology-derived proteins as active substance [1]. It is anticipated that within the next $5-10$ years, $50 \%$ of all the drugs in development will be biological in origin. This increase in research and development of such molecules are due to their efficacy in disrupting the exact pathophysiology of the disease. Since the last decade, biologics have become the mainstay of treatment of many retinal vascular pathologies. They have transformed the visual outcomes of such diseases and have reduced the disease-related blindness. Currently, the anti-VEGF molecules being administered intravitreally are bevacizumab, ranibizumab, aflibercept and brolucizumab. Bevacizumab is being used off label in monthly dosing regimen, ranibizumab is the first FDA-approved anti-VEGF molecule for ophthalmic use in monthly dosing regimen, while aflibercept is approved by FDA for $\mathrm{q} 8 \mathrm{w}$ dosing. Hinderances in instituting and maintaining biological therapy in ophthalmology are frequent visit to hospitals, repeated injections and the cost of the drug itself. The financial burden is found to be the major cause of patient non-compliance to monthly dosing regimen. In order to increase the dosing interval and thus reducing the cumulative cost of treatment, various regimens have been tried, most successful being Pro Re Nata and treat and extend approaches. These treatment protocols have

$\triangle$ Ashish Sharma

drashish79@hotmail.com

1 Department of Vitreoretina, Lotus Eye Hospital and Institute, Coimbatore, TN, India

2 Department of Ophthalmology; University Vita-Salute, Scientific Institute San Raffaele, Milano, Italy

3 Division of Ophthalmology, Tel Aviv Sourasky Medical Center and Sackler Faculty of Medicine, Tel Aviv University, Tel Aviv, Israel

4 Gavin Herbert Eye Institute, University of California, Irvine, CA, USA though been shown to be inefficient in maintaining the visual gains in long term follow-up when compared with monthly dosing regimen [2]. Recently, there has been advancement in development of newer anti-VEGF agents which inherently increase the dosing interval from 4 weeks to $8-12$ weeks, and thus can reduce cost. But none of the methods to reduce the financial burden is as realistic as biosimilars [3-5].

Biosimilars are the near-identical copies of the innovator biologic or reference product which do not have a significant difference in terms of efficacy or safety in clinical use [6]. These biosimilars have potential to reduce cost of treatment up to $30 \%$ without compromising on the dosing interval and are validated to be used in all the indications for which the reference product is approved for administration. The regulatory authorities have issued comprehensive guidelines for the development and approval of biosimilars across the globe to maintain the safety index of the molecule. These guidelines mandate the biosimilars to be proven similar to the reference product in large number of analytical studies about the structure and few clinical trials to assess the safety among the human subjects [7]. The reduced number of clinical trials, lesser investment into the research and development of molecule due to the presence of a reference product are few factors which help to reduce the cost of the drug. The presence of lower-cost options is also anticipated to increase the market competition, which also will lead to reduction in cost of therapy.

Biosimilars are increasing in number. FDA has approved 11 biosimilars till date for use in various diseases. Razumab (Intas Pharmaceutical Ltd, Ahmedabad, GJ, India) is the first, and till now the only biosimilar to ranibizumab that has been approved for ophthalmic use [8]. It is approved for use in India by the drug controller general of India. The biosimilars were anticipated to be adopted by healthcare professionals and patients for their reduced cost. They, however, have faced resistance due to widely popular perception of biosimilar being generic versions of innovator molecules. That brings in a nocebo 
(negative perception) effect among the care providers and patients. The negative perception may increase reporting of minor adverse events such as low-grade inflammation, which usually is overlooked in therapy involving innovator molecule. Our group has evaluated the switching of patients from ranibizumab to its biosimilar (razumab) in different indications (Ci-DME, BRVO, CRVO and n-AMD), and have found to maintain safety and efficacy. Furthermore our data revealed no signs of immunogenicity after switching (Unpublished data under review). Furthermore no differences were observed between size variants and potency between original and biosimilar ranibizumab [9]. In order to combat such nocebo surrounding the drug and to sensitize the patients and healthcare professionals about the potential benefits of biosimilars while highlighting the regulatory guidelines that govern the biosimilar development, FDA has increasingly been issuing education materials for the same [10]. FDA conducted a survey where healthcare professionals revealed loss of clarity on various terms that are used to define biosimilars, switching, interchangeability and immunogenicity. They have launched a dedicated web portal to provide one-stop answer to all such biosimilar related queries [11].

Several other biosimilars for ophthalmic use are in pipeline across the globe for development with their launch on the horizon [6]. The development of such biosimilars are strictly regulated by their concerned authorities, thus are proven for their safety and efficacy [7]. These regulatory bodies, akin to FDA should initiate outreach programmes and create education materials to sensitize the providers and patients. Professional societies should also take an initiative to understand such molecules, facilitate dialogues between their members, manufacturers and regulatory authorities to remove the nocebo effect. Widespread adoption of biosimilars will reduce the healthcare cost, and thus can also help to bring patients who currently cannot afford the treatment into the ambit, which also will reduce the disease burden itself. It can also help to reduce the cost for insurance providers like NHS, who are now burdened with rising cost of prescription drugs.

\section{Compliance with ethical standards}

Conflict of interest The authors declare that they have no conflict of interest.

Publisher's note Springer Nature remains neutral with regard to jurisdictional claims in published maps and institutional affiliations.

\section{References}

1. US FDA. Biological Product Definitions. FDA. https://www.fda. gov/media/108557/download. Accessed 25 Oct 2019.

2. The Vision Academy Steering Committee, Lanzetta P, Loewenstein A. Fundamental principles of an anti-VEGF treatment regimen: optimal application of intravitreal anti-vascular endothelial growth factor therapy of macular diseases. Graefes Arch Clin Exp Ophthalmol. 2017;255:1259-73.

3. Dugel PU, Koh A, Ogura Y, Jaffe GJ, Schmidt-Erfurth U, Brown DM, et al. HAWK and HARRIER: Phase 3, multicenter, randomized, double-masked trials of brolucizumab for neovascular age-related macular degeneration. Ophthalmology. 2019; pii: S0161-6420(18)33018-5.

4. Sharma A, Kumar N, Kuppermann BD, Loewenstein A, Bandello F. Brolucizumab: is extended VEGF suppression on the horizon? Eye (Lond). 2019. https://doi.org/10.1038/s41433-019-0582-0.

5. Sharma A, Kumar N, Kuppermann BD, Bandello F. Abicipar pegol: the non-monoclonal antibody anti-VEGF. Eye (Lond). 2019. https://doi.org/10.1038/s41433-019-0607-8.

6. Sharma A, Reddy P, Kuppermann BD, Bandello F, Loewenstein A. Biosimilars in ophthalmology: is there a big change on the horizon? OPTH. 2018;12:2137-43.

7. Sharma A, Kumar N, Kuppermann BD, Bandello F, Loewenstein A. Understanding biosimilars and its regulatory aspects across the globe: an ophthalmology perspective. Br J Ophthalmol. 2019; pii: bjophthalmol-2019-314443.

8. Sharma A, Kumar N, Kuppermann B, Francesco B, Lowenstein A. Ophthalmic biosimilars: lessons from India. Indian J Ophthalmol. 2019;67:1384.

9. Griaud F, Winter A, Denefeld B, Lang M, Hensinger H, Straube $\mathrm{F}$, et al. Identification of multiple serine to asparagine sequence variation sites in an intended copy product of LUCENTIS $^{\circ}$ by mass spectrometry. MAbs 2017;9:1337-48.

10. Center for Drug Evaluation and Research. Health Care Provider Materials. US FDA. 2019. http://www.fda.gov/drugs/biosimilars/ health-care-provider-materials. Accessed 24 Oct 2019.

11. Center for Drug Evaluation and Research. Biosimilars. US FDA. 2019. http://www.fda.gov/drugs/therapeutic-biologics-applicationsbla/biosimilars. Accessed 24 Oct 2019. 